\title{
Navigation in a small world
}

\section{It is easier to find short chains between points in some networks than others.}

p he small-world phenomenon - the principle that most of us are linked by short chains of acquaintances - was first investigated as a question in sociolo$\mathrm{gy}^{1,2}$ and is a feature of a range of networks arising in nature and technology ${ }^{3-5}$. Experimental study of the phenomenon ${ }^{1}$ revealed that it has two fundamental components: first, such short chains are ubiquitous, and second, individuals operating with purely local information are very adept at finding these chains. The first issue has been analysed ${ }^{2-4}$, and here I investigate the second by modelling how individuals can find short chains in a large social network.

I have found that the cues needed for discovering short chains emerge in a very simple network model. This model is based on early experiments ${ }^{1}$, in which source individuals in Nebraska attempted to transmit a letter to a target in Massachusetts, with the letter being forwarded at each step to someone the holder knew on a first-name basis. The networks underlying the model follow the 'small-world' paradigm ${ }^{3}$ : they are rich in structured short-range connections and have a few random long-range connections.

Long-range connections are added to a two-dimensional lattice controlled by a clustering exponent, $\alpha$, that determines the probability of a connection between two nodes as a function of their lattice distance (Fig. 1a). Decentralized algorithms are studied for transmitting a message: at each step, the holder of the message must pass it across one of its short- or long-range connections; crucially, this current holder does not know the long-range connections of nodes that have not touched the message. The primary figure of merit for such an algorithm is its expected delivery time $T$, which represents the expected number of steps needed to forward a message between a random source and target in a network generated according to the model. It is crucial to constrain the algorithm to use only local information - with global knowledge of all connections in the network, the shortest chain can be found very simply ${ }^{6}$.

A characteristic feature of small-world networks is that their diameter is exponentially smaller than their size, being bounded by a polynomial in $\log N$, where $N$ is the number of nodes. In other words, there is always a very short path between any two nodes. This does not imply, however, that a decentralized algorithm will be able to discover such short paths. My central finding is that there is in fact a unique value of the exponent $\alpha$ at which this is possible.

When $\alpha=2$, so that long-range connec- tions follow an inverse-square distribution, there is a decentralized algorithm that achieves a very rapid delivery time; $T$ is bounded by a function proportional to $(\log N)^{2}$. The algorithm achieving this bound is a 'greedy' heuristic: each message holder forwards the message across a con-
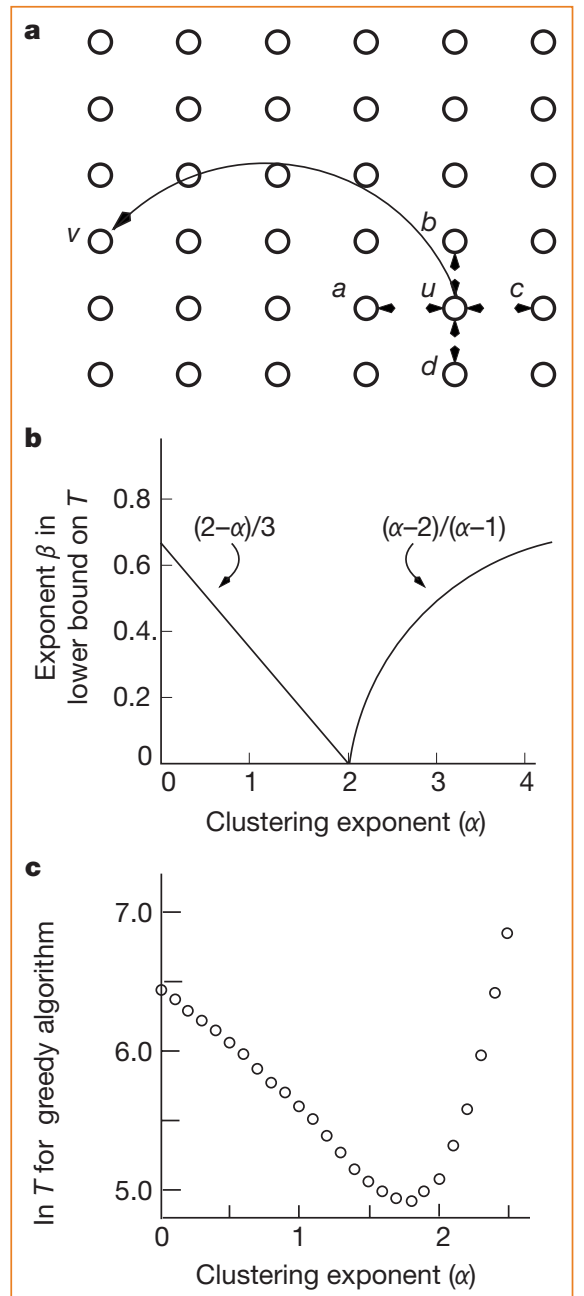

Figure 1 The navigability of small-world networks. a, The network model is derived from an $n \times n$ lattice. Each node, $u$, has a shortrange connection to its nearest neighbours $(a, b, c$ and $d)$ and a long-range connection to a randomly chosen node, where node $v$ is selected with probability proportional to $r^{-\alpha}$, where $r$ is the lattice ('Manhattan') distance between $u$ and $v$, and $\alpha \geqslant 0$ is a fixed clustering exponent. More generally, for $p, q \geqslant 1$, each node $u$ has a short-range connection to all nodes within $p$ lattice steps, and $q$ long-range connections generated independently from a distribution with clustering exponent $\alpha$. b, Lower bound from my characterization theorem: when $\alpha \neq 2$, the expected delivery time $T$ of any decentralized algorithm satisfies $T \geqslant c n^{\beta}$, where $\beta=(2-\alpha) / 3$ for $0 \leqslant \alpha<2$ and $\beta=(\alpha-2) /(\alpha-1)$ for $\alpha>2$, and where $c$ depends on $\alpha, p$ and $q$, but not $n$. c, Simulation of the greedy algorithm on a 20,000 $\times 20,000$ toroidal lattice, with random long-range connections as in $\mathbf{a}$. Each data point is the average of 1,000 runs. nection that brings it as close as possible to the target in lattice distance. Moreover, $\alpha=2$ is the only exponent at which any decentralized algorithm can achieve a delivery time bounded by any polynomial in $\log N$ : for every other exponent, an asymptotically much larger delivery time is required, regardless of the algorithm employed (Fig. 1b).

These results indicate that efficient navigability is a fundamental property of only some small-world structures. The results also generalize to $d$-dimensional lattices for any value of $d \geqslant 1$, with the critical value of the clustering exponent becoming $\alpha=d$. Simulations of the greedy algorithm yield results that are qualitatively consistent with the asymptotic analytical bounds (Fig. 1c).

In the areas of communication networks ${ }^{7}$ and neuroanatomy ${ }^{8}$, the issue of routing without a global network organization has been considered; also in social psychology and information foraging some of the cues that individuals use to construct paths through a social network or hyperlinked environment have been discovered ${ }^{9,10}$. Although I have focused on a very clean model, I believe that a more general conclusion can be drawn for small-world networks - namely that the correlation between local structure and long-range connections provides critical cues for finding paths through the network.

When this correlation is near a critical threshold, the structure of the long-range connections forms a type of gradient that allows individuals to guide a message efficiently towards a target. As the correlation drops below this critical value and the social network becomes more homogeneous, these cues begin to disappear; in the limit, when long-range connections are generated uniformly at random, the result is a world in which short chains exist but individuals, faced with a disorienting array of social contacts, are unable to find them.

\section{Jon M. Kleinberg}

Department of Computer Science, Cornell

University, Ithaca, New York 14853, USA

1. Milgram, S. Psychol. Today 1, 61-67 (1967).

2. Kochen, M. (ed.) The Small World (Ablex, Norwood, NJ, 1989).

3. Watts, D. \& Strogatz, S. Nature 393, 440-442 (1998).

4. Albert, R. et al. Nature 401, 130-131 (1999).

5. Adamic, L. in Proc. 3rd European Conference on Digital Libraries (eds Abiteboul, S. \& Vercoustre, A.-M.) 443-452 (Springer Lecture Notes in Computer Science, Vol. 1696, Berlin, 1999).

6. Cormen, T., Leiserson, C. \& Rivest, R. Introduction to Algorithms (McGraw-Hill, Boston, 1990).

7. Peleg, D. \& Upfal, E. J. Assoc. Comput. Machinery 36, 510-530 (1989).

8. Braitenberg, V. \& Schüz, A. Anatomy of the Cortex (Springer, Berlin, 1991).

9. Killworth, P. \& Bernard, H. Social Networks 1, 159-192 (1978) 10. Pirolli, P. \& Card, S. Psychol. Rev. 106, 643-675 (1999). 